May 13, 2018

\title{
Cooling rate dependence of the internal structure of a Lennard-Jones glass
}

\author{
Katharina Vollmayn, Walter Kobli and Kurt Binder \\ Institut für Physik, Johannes Gutenberg-Universität, Staudinger Weg 7, D-55099 Mainz, \\ Germany
}

\begin{abstract}
Using molecular dynamics computer simulations we investigate the dependence of bulk properties of a Lennard-Jones glass on the cooling rate with which the glass was produced. By studying the clusters formed by the nearest neighbor shells of the particles, we show that the internal structure of the glass depends on the cooling rate and that the cooling rate dependence of the enthalpy of the glass can be understood by the change of this internal structure.
\end{abstract}

PACS numbers: 61.20.Lc, 61.20.Ja, 02.70.Ns, 64.70.Pf 
If a material is cooled fast enough from a high temperature liquid state to a low temperature solid state, the crystallization of the substance at or slightly below its melting point can be avoided and the end product will be an amorphous solid, i.e. a glass. Since this so-called glass transition is essentially the falling out of equilibrium of the system because the typical time scale of the experiment is exceeded by the typical time scale of the relaxation times of the system, the resulting glass can be expected to depend on the way the glass was produced, e.g., on the cooling rate of the sample or the particulars of the cooling schedule. Such a dependency of the density of the glass or of the glass transition temperature has indeed been found in experiments [1,2] and in computer simulations [3]. Although the presence of such a cooling rate dependence is therefore well established (although, e.g., Speedy reports that no dependency of the properties of a hard sphere glass on the quench rate could be found [4]), the exact reason for this dependence is still not known. Is it mainly that the global arrangement of the particles depends on the cooling rate, or is this global arrangement essentially cooling rate independent (of course only in a statistical sense) and it is only that the positions of the particles within this arrangement depend on the cooling rate? E.g., using a molecular dynamics computer simulation Jónsson and Andersen have found that a binary Lennard-Jones glass has at low temperatures clusters of interpenetrating icosahedra that percolate throughout the sample [5]. Thus one might wonder whether this whole percolating structure shows a cooling rate dependence or whether the topology of the structure is basically independent of the cooling rate and it is only the details of the positions of the particles within this structure that give rise to the cooling rate dependence of the various macroscopic properties of the glass. Since computer simulations are a most useful tool to study the full microscopic details of such systems they should allow to gain some insight into this question and in this Letter we report some of our findings of our investigations in this direction.

The model we are considering is a binary mixture of Lennard-Jones particles (type A and type B), both of them having the same mass $m$. The interaction between particles of type $\alpha$ and $\beta(\alpha, \beta \in\{A, B\})$ is given by $V_{\alpha \beta}(r)=4 \epsilon_{\alpha \beta}\left[\left(\sigma_{\alpha \beta} / r\right)^{12}-\left(\sigma_{\alpha \beta} / r\right)^{6}\right]$ with $\epsilon_{A A}=1.0, \epsilon_{A B}=1.5, \epsilon_{B B}=0.5, \sigma_{A A}=1.0, \sigma_{A B}=0.8$ and $\sigma_{B B}=0.88$. In order to decrease the computational load $V_{\alpha \beta}(r)$ was truncated and shifted at $r=2.5 \sigma_{\alpha \beta}$. This potential has been used previously to study the dynamics of strongly supercooled liquids and thus it is known that this system is a good glass former, i.e. not prone to crystallization [6].

In the following all results are given in reduced units with $\sigma_{A A}$ the unit of length, $\epsilon_{A A}$ the unit of energy and $\left(m \sigma_{A A}^{2} / 48 \epsilon_{A A}\right)^{1 / 2}$ the unit of time. Using the constant pressure algorithm by Andersen [7] the equations of motion were integrated with a step size of 0.02 and an external presssure of 1.0. The system was equilibrated at a high temperature $T_{0}=$ 2.0 where it is in a liquid state. Subsequently it was coupled to a stochastic heat bath whose temperature $T_{b}$ was decreased linearly in time, i.e. $T_{b}(t)=T_{0}-\gamma t$, where $\gamma$ is the cooling rate. This quench was done until the temperature of the heat bath was zero. Using a steepest descent procedure, the resulting configuration of particles was relaxed to its nearest metastable minimum in configuration space and the so obtained configurations were subsequently analyzed. Note that during this steepest descent procedure also the volume of the system was allowed to relax. The range of cooling rates we investigated was $3.1 \cdot 10^{-6} \leq \gamma \leq 2.0 \cdot 10^{-2}$. The number of A and B particles was 800 and 200, respectively. In order to improve the statistics of our results, we performed for each value of $\gamma$ ten different 
runs and averaged over these runs.

In Fig. 1 we show $H_{f}(\gamma)$, the final enthalpy of the system after having quenched the system to $T=0$ with a cooling rate $\gamma$. We see that a variation of $\gamma$ over about four decades leads to a change in $H_{f}(\gamma)$ of only $1-2 \%$. The smallness of this change shows that in order to investigate such cooling rate dependencies it is important to vary $\gamma$ over several orders of magnitude and to use either fairly large systems or to average over many small ones.

Qualitatively the same behavior as in the case $H_{f}(\gamma)$ was found also in other quantities such as the density, the coordination number of the particles or the radial distribution functions. In all cases the effect of the variation of the cooling rate was to give rise to a change of a few percent of the quantity investigated [8]. The size of these cooling rate effects is comparable with the one found in real experiments, in that, e.g., Brüning et al found that the density of $\mathrm{B}_{2} \mathrm{O}_{3}$ also changes by about $1 \%$ when the cooling rate is varied over three decades [2].

Now that we have demonstrated that our system shows, similar to experiments, a cooling rate dependence of various bulk properties of the glass, we can investigate the problem mentioned in the introduction, namely whether these properties depend on the cooling rate because the whole internal structure of the glass depends on the cooling rate or whether this structure is essentially cooling rate independent and the bulk properties of the system change only because the particles re-adjust their position by small amounts without that the properties of this global structure are changed.

In order to address this question we investigated the cooling rate dependence of the properties of the nearest neighbor shells of the particles. The nearest neighbor shell of a particle of type $\alpha \in\{A, B\}$ was defined to be the set of all those particles that have a distance $r$ which was less than the location of the minimum in the corresponding radial distribution function $g_{\alpha \beta}(r)$. For the sake of clarity we will discuss in the following only the nearest neighbor shells of the A particles, i.e. of the majority species. However, similar results have also been found for the $\mathrm{B}$ particles.

We define $N_{A_{z, \mu}}$ to be the number of A particles that have $z$ nearest neighbors with $\mu$ of them being a B particle. In the following we will call the collection of particles given by the central particle and its nearest neighbor shell a "cluster". The energy $E_{c}$ of such a cluster is defined to be the sum over all the interactions of the particles within this cluster. In Fig. 2 we show the dependence of $E_{c}$ on the cooling rate. In order not to crowd the figure too much we show only the curves for a subset of the clusters we found, but the curves for the clusters not displayed show qualitatively the same $\gamma$ dependence as those shown. Three observations can be made from this figure: 1) The energy of all clusters is decreasing with decreasing $\gamma$. 2) For every given type of cluster the difference in $E_{c}$ between the fastest and the slowest cooling rate is on the order of 1\%. 3) For a given cooling rate the difference between the energy of the various clusters is generally much larger than $1 \%$.

Since the enthalpy of the system is related to the weighted sum of the energy of the clusters and since we have seen in Fig. 1 that the former shows a change of about 1\% when the cooling rate is varied over several decades, one might conclude from points 1) and 2) that the cooling rate dependence of the enthalpy of the system is given entirely by the cooling rate dependence of the clusters. However, before this conclusion can be drawn it is of course necessary to investigate whether the frequency of the clusters of the various types does not show a significant $\gamma$ dependence, since the third point shows that such an effect might be 
important.

In Fig. 3 we show $P_{A_{z, \mu}}$, the probability to find a cluster of type $A_{z, \mu}$, for all those combinations of $z$ and $\mu$ for which this probability is not too small. From this figure we recognize that there are clusters whose corresponding $P_{A_{z, \mu}}$ is essentially independent of the cooling rate, such as $A_{13,2}$, and that there are other clusters, marked with symbols, whose $P_{A_{z, \mu}}$ changes significantly with $\gamma$. Among these latter types of clusters there are some whose $P_{A_{z, \mu}}$ changes by as much as $20 \%$ (e.g. $A_{12,1}$ ). Thus if one takes into account that the difference between the energy between the various clusters is usually significantly larger than $1 \%$ (see point 3) and Fig. 2) such a change in $P_{A_{z, \mu}}$ can lead to a change in the enthalpy of the system that is significantly larger than the one that comes from the change of the energy of the cluster itself.

In order to demonstrate this effect clearer we show in Fig. $4 P\left(E_{c}\right)$, the probability that a cluster has an energy $E_{c}$, versus $E_{c}$ for all cooling rates investigated. We see that this distribution function shows various peaks, each of which can be identified with particular types of clusters. From this figure we also recognize that the main difference between the distribution for the fastest cooling rate (bold dashed curve) and the distribution for the slowest cooling rate (bold solid curve) is that the height of these peaks changes. Since the position of the peaks is given by the mean energy of the corresponding cluster and since we find that this position is essentially independent of $\gamma$ we thus come to the conclusion that the enthalpy of the system shows a cooling rate dependence because the frequency of the various clusters shows a strong dependence on $\gamma$ and not because the energy of the individual clusters is changing.

In this Letter we have focused on investigating the cooling rate dependence of the enthalpy of the system. Very similar results have also been obtained for the cooling rate dependence of the density [8]. Also for this quantity we found that it is the $\gamma$ dependence of the distribution function for the clusters rather than the individual properties of the clusters themselves that gives rise to the $\gamma$ dependence of the density. Thus we can summarize this work by saying that the cooling rate effects observed in structural glass formers follow from the fact that the internal structure of the glass depends on the cooling rate and not that there exists an internal structure that is independent of the cooling rate and that the particles within this structure show a cooling rate dependence. Although our findings apply, strictly speaking, only to the system investigated here, it can be expected that the same or at least a similar mechanism is present also in other structural glass formers and thus our conclusions should be valid for a relatively large class of glass formers.

Acknowledgements: We thank C. A. Angell for valuable discussions. K. V. thanks Schott Glaswerke, Mainz, for financial support. Part of this work was done on the computer facilities of the Regionales Rechenzentrum Kaiserslautern. 


\section{REFERENCES}

† Electronic mail: vollmayr@moses.physik.uni-mainz.de

* Corresponding author. Electronic mail: kob@moses.physik.uni-mainz.de http://www.cond-mat.physik.uni-mainz.de/ kob/home_kob.htm]

[1] see, e.g., H. N. Ritland, J. Amer. Ceram. Soc. 37, 370 (1954); C. Y. Yang, D. E. Sayers and M. A. Paesler, Phys. Rev. B 36, 8122 (1987); C. T. Limbach and U. Gonser, J. Non-Cryst. Solids 106, 399 (1988); G. P. Johari, A. Hallbrucker and E. Mayer, J. Phys. Chem. 93, 2648 (1989); R. Brüning and K. Samwer, Phys. Rev. B 46, 11318 (1992).

[2] R. Brüning and M. Sutton, Phys. Rev. B 49, 3124 (1994).

[3] J. R. Fox and H. C. Andersen, J. Phys. Chem. 88, 4019 (1984); H. Miyagawa and Y. Hiwatari, Phys. Rev. A 40, 6007 (1989); S. K. Lai and M. S. Lin, J. Non-Cryst. Solids 117/118, 907 (1990).

[4] R. Speedy, Mol. Phys. 83, 591 (1994).

[5] H. Jónsson and H. C. Andersen, Phys. Rev. Lett. 60, 2295 (1988).

[6] W. Kob and H. C. Andersen, Phys. Rev. Lett. 73, 1376 (1994); Phys. Rev. E 51, 4626 (1995); ibid. 52, xxxx (1995).

[7] H. C. Andersen, J. Chem. Phys. 72, 2384 (1980).

[8] K. Vollmayr, W. Kob and K. Binder (preprint). 


\section{FIGURES}

Fig. 1: Final enthalpy $H_{f}$ versus the cooling rate $\gamma$.

Fig. 2: $E_{c}$, the energy of a cluster, for a few selected types of clusters around A particles versus the cooling rate $\gamma$. See text for the meaning of the two indices identifying the type of the cluster.

Fig. 3: $P_{A_{z, \mu}}$, the probability to find a cluster of type $A_{z, \mu}$, versus the cooling rate $\gamma$. Only the most frequent types of clusters are shown. See text for the meaning of the two indices identifying the type of the cluster.

Fig. 4: $P\left(E_{c}\right)$, the probability that a cluster has an energy $E_{c}$, versus $E_{c}$ for all cooling rates investigated. The bold solid and dashed lines correspond to the slowest and fastest cooling rates, respectively. 